\title{
A Statistical Study of Spectroscopic Binaries Containing A Late-Type Giant Star
}

\author{
H.M. J. Boffin ${ }^{1}$ \\ Institut d'Astrophysique \\ Universite Libre de Bruxelles
}

1 Boursier I.R.S.I.A.

Binarity seems to be a feature shared by various classes of Peculiar Red Giants (PRG). This observational fact has led to the general agreement that those stars result from a mass transfer originating from an asymptotic giant branch companion star.

To discover which of the two mass exchange scenarios (1.e.. a stellar wind or Roche lobe overflow) actually operates necessitates a careful study of the PRG's orbits. That study should compare the orbital parameters of PRG's with those of normal red giants. Understanding the behaviour of so-called normal stars is thus a pre-requisite to the study of the distribution of the PRG's orbital elements distribution. To this end, have we constructed, from the available literature, a catalogue of 195 spectroscopic binaries containing at least one late-type giant. From a statistical study of this catalogue can we extract some data of high interest in view of the comparison with a sample of PRGs orbits.

We deduce the following mean values from our catalogue: $\langle P\rangle=1098 \pm 232$ days, $\langle e\rangle=0.205 \pm 0.015,\left\langle e^{2}\right\rangle=0.09 \pm 0.01$, $\langle f(M)\rangle=0.124 \pm 0.015 \mathrm{M}_{\odot}$.

The well-known orbital period-eccentricity correlation (physically (un)meaningful?) is also present in our sample and can be written $\langle e\rangle \approx 0.16 \log \mathrm{P}-0.16$.

Other statistical properties are given, namely the distributions of eccentricity, period and mass function. We also at tempt to determine the distribution of the mass ratio in such systems. 